\title{
Properties of Cartilage on Micro- and Nanolevel
}

\author{
Sergei A. Chizhik, ${ }^{1}$ Krzysztof Wierzcholski, ${ }^{2}$ Anastasia V. Trushko, ${ }^{1}$ \\ Mariya A. Zhytkova, ${ }^{1}$ and Andrzej Miszczak ${ }^{2}$ \\ ${ }^{1}$ Heat and Mass Transfer Institute, National Academy of Sciences of Belarus, 15. P.Brovki, Minsk 220072, Belarus \\ ${ }^{2}$ Department of Basic Engineering, Gdynia Maritime University, 83 Morska, 81-225 Gdynja, Poland
}

Correspondence should be addressed to Mariya A. Zhytkova, mzhytkova@gmail.com

Received 15 December 2009; Revised 2 November 2010; Accepted 25 November 2010

Academic Editor: J. Paulo Davim

Copyright ( 2010 Sergei A. Chizhik et al. This is an open access article distributed under the Creative Commons Attribution License, which permits unrestricted use, distribution, and reproduction in any medium, provided the original work is properly cited.

\begin{abstract}
Results of investigation of the elastic modulus for cartilage tissue using a technique of micro- and nanoindentation performed with help of an atomic force microscope are presented. SEM and AFM methods were applied to visualize a topography of surface layers of the entire cartilage and as well as its slices and thus to reveal features of the collagen fibers orientation. The technique used for a quantitative evaluation of the elastic modulus under compression against a ball microindenter (curvature radius - 350 micron) and a nanoindenter $(30 \mathrm{~nm})$ is described. It was shown that the cartilage behavior is highly stabile under the load if the entire composite structure of cartilage tissue is engaged into the deformation process. Tribological characteristics were investigated using the ball indenter oscillated by a tuning fork. Dependence of the friction coefficient from applied loads was obtained that revealed strong influence of an interstitial fluid on friction properties. Friction coefficient of a rat cartilage tissue as 0.08 was obtained using a developed plant prototype for tribological measurements based on the AFM construction.
\end{abstract}

\section{Introduction}

Exclusively high reliability and durability of the synovial joints being natural units for load and friction transmission are defined by their complex structure that ensures both high load capacity and low friction $[1,2]$. Unique properties of the joint cartilage attract great attention of engineers and scientists as superior though yet unreachable prototype for material science solutions in designing friction assemblies for machine and robot building as well as for artificial joints. The mechanisms of the deformation and friction of the synovial joint cartilage are widely described in scientific literature; however, there is still no common and universally recognized friction theory [3-5].

Complex structure of cartilage tissue was studied in detail quite well. It represents a porous matrix of collagen fibers arranged in a special pattern that is filled with a liquid containing proteoglycans with electrostatically charged elements that provide osmotic pressure in the tissue and also small amount of hyaluronic acid exhibiting high protective and antifrictional properties (Figure 1). Microscopic methods showed explicit hierarchical (multiscale) and anisotropic structure of the collagen framework (Figure 1(a)) having main structural elements of nanometer size (diameter of collagen fibers $10-100 \mathrm{~nm}$ ). Collagen fibers in the surface layer are arranged parallel to the cartilage surface (lateral arrangement) while in the inner layers they are oriented almost perpendicular to the bone and cartilage surface, that is, have radial arrangement (Figure 1(b)).

In unloaded joint cartilage, strain of the collagen network is balanced by the osmotic pressure of proteoglycans. Hence, collagen framework carries out structuring and stabilizing function with respect to the proteoglycans and water and also regulates degree of the cartilage tissue hydration; it possesses high elasticity with respect to stretching forces.

We can cite a series of works [6-9] devoted to the study of elastic and viscoelastic deformation of joint cartilage under its compression in millimeter range. Elasticity of cartilage tissue in macrovolume can be estimated even under clinic conditions during arthroscopy $[10,11]$. Generalizing analysis of results obtained in various works shows substantial difference in the measured elastic modulus. That is explained by explicit dependence of cartilage viscoelastic properties on the loading duration and structure heterogeneity under 


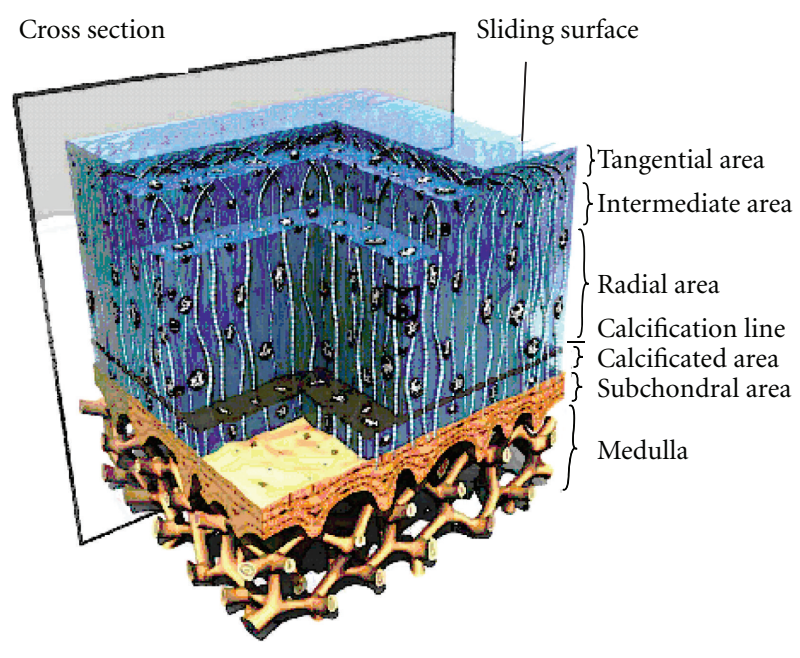

(a)

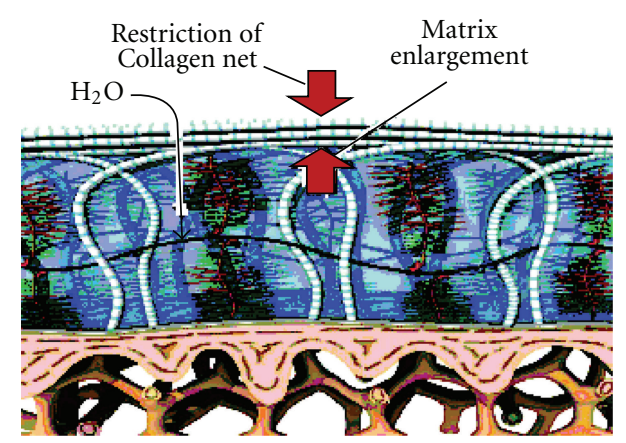

(b)

Figure 1: Structure of cartilage tissue: (a) on microlevel with emphasized heterogeneity zones; (b) arrangement of nanosized components.

the stipulation that the experiments applied short-term as well as long-term compression, stretching, and shear loads.

For complete understanding of the cartilage mechanical behavior, it is important to measure mechanical properties of the main components of its structure, for instance, collagen fibers having diameter of several tens of nanometers. It is interesting to consider behavior of the fibers embedded into the entire cartilage structure in dependence on their orientation in the framework. Quantitative evaluation of joint cartilage elasticity on nanolevel still remains a nontrivial procedure. In all probability, the most acceptable way for such evaluation can be application of a force spectroscopy procedure of the atomic force microscopy [12]. The procedure applies minimum compression load beginning from several nanonewtons to the areas restricted by several square nanometers that is performed by touching the studied surface feature with a nanoindenter with the curvature radius of $10-50 \mathrm{~nm}$.

The purpose of the work is the qualitative analysis of cartilage material deformation on nanolevel at the collagen fiber orientation along and across direction of the local loading and the result comparison with macrolevel deformation when the entire integral structure of cartilage tissue is engaged into the process. It is supposed that the investigation will make it possible to specify the mechanism of the cartilage nanostructure effect on the mechanical properties and to explain the nature of its high load capacity.

The additional idea of the work was to investigate a role of the interstitial fluid in friction processes and to create some prototype to measure friction in situ.

\section{Object and Technique of the Investigation}

As objects for the investigation, radial and tangential slices of pork femur cartilage as well as block samples of cartilage structure were implemented as objects for the investigation. The samples were prepared as follows.

Initially, two platelets of cartilage sizing $5 \times 5 \times 2 \mathrm{~mm}$ were cut from femur surface with sharp blade. Then radial and tangential slices sizing $\sim 5 \times 2 \times 0.5$ were cut off, one of the platelets with the same blade. The finished samples were stored for certain time at temperature $-12^{\circ} \mathrm{C}$.

Surface structure of the cartilage samples were studied by the scanning electron (SEM) and the atomic force microscopy (AFM). In the latter case, we had an opportunity to visualize spatial topography that correlated with the material collagen structure in surface layer and to measure directly the sample surface roughness parameters.

For measurement of the sample mechanical properties, AFM NT-206 (Microtestmachines, Belarus) was applied [13]. Based on the conventional AFM system (Figure 2(a)), static force spectroscopy procedure was implemented.

At the force spectroscopy, probe tip mounted on a free end of flexible microcantilever is positioned over the studied feature on the sample surface with high accuracy (up to $10 \mathrm{~nm}$ ). Then the sample is moved vertically toward the probe tip under the step control better than $1 \mathrm{~nm}$. After reaching the preset position, the sample is then withdrawn off the probe tip. While realizing the static force spectroscopy procedure, the cantilever is not driven into oscillations, and the measuring system plots the microcantilever deflection $Z_{\text {defl }}$ according to the relative vertical position $Z_{\text {pos }}$ of the fixed probe end and the sample. Deflection of the cantilever with spring constant $k$ after entering probe tip into contact with the sample surface defines the applied indentation load

$$
P=k Z_{\text {defl }}
$$

Deformation depth of the material in the indentation point is

$$
H=Z_{\text {pos }}-Z_{\text {defl }} \text {. }
$$

Elastic modulus is calculated under the condition of equilibrium between the deflected probe cantilever and elastic deformation of the sample material by the probe tip (Figure 2(b)). Taking into account expressions (1) and (2) and supposing that probe tip material is much more rigid in comparison with the measured sample material and the probe tip has spherical shape, from the Hertzian model we 


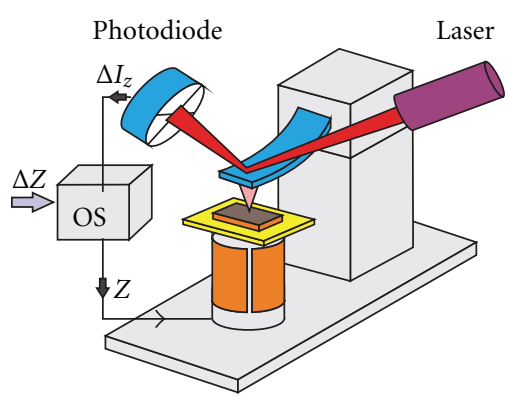

(a)

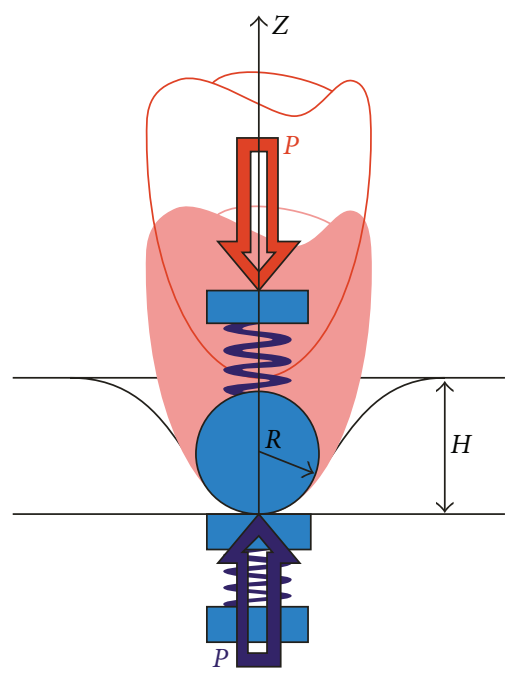

(b)

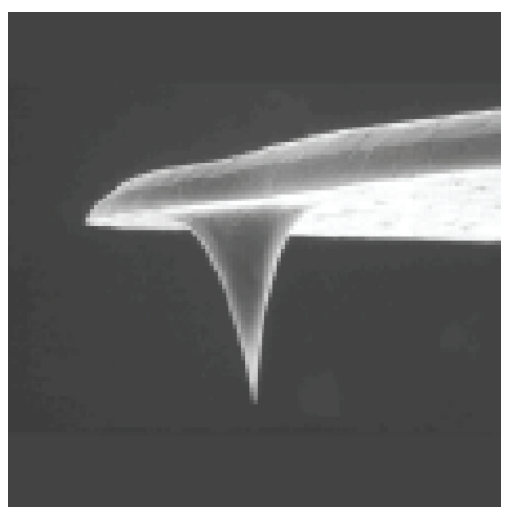

(c)

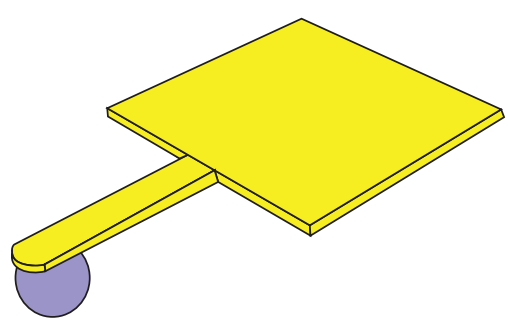

(d)

FIgurE 2: Scheme of the indentation with AFM: (a) schematic diagram of AFM with laser-beam system of the cantilever deflection detection; (b) geometry set of the elastic modulus calculation; (c) AFM probe tip used as indenter for nanometer-level measurement; (d) ball indenter for macrolevel measurement. can derive an expression for local Young modulus at the point $i$ of the studied surface

$$
E_{i}=\frac{3}{4}\left(1-v^{2}\right) \frac{k}{R^{1 / 2}} \frac{Z_{\text {defl }}}{\left(Z_{\text {pos }}-z_{\text {defl }}\right)^{3 / 2}} .
$$

Here $R$ is tip curvature radius, and $v$ is Poisson's ratio that for cartilage was received as 0.5 .

The AFM design allows implementing two types of measurements differing with type of the used indenting probe. Nanolevel measurements were performed with the commercially available silicon cantilever CSC38 (Mikromasch) having the tip curvature radius $R=30 \mathrm{~nm}$ and spring constant $k=0,08 \mathrm{~N} / \mathrm{m}$ (Figure 2(b)). For microlevel measurements, we used microfabricated probe consisting of steel cantilever having spring constant $k=11 \mathrm{~N} / \mathrm{m}$ with bearing ball of $R=350$ micron attached on its free end that served as indenter. In the first case, contact area makes several nanometers and is much less than the collagen fiber cross-section. In the second case, contact area was estimated equaling several micrometers that significantly exceeds characteristic dimensions of cartilage structure nanoelements.

Tribological properties were evaluated by the means of spherical indenter that is oscillated parallel to the investigated surface. The indenter is geared by a tuning fork that is fixed in the AFM holder.

Friction force and friction coefficient are calculated as follows:

$$
F=\frac{\pi k}{4 Q}\left(A_{0}-A\right)
$$

where $k$ is the tuning fork stiffness coefficient, $A_{0}$ is the tuning fork oscillation amplitude in case of surface contact absence, and $A$ is the oscillation amplitude in case of contact. Friction coefficient is evaluated as $K=F / F_{\text {Load }}$.

Here $F$ is friction force, and $F_{\text {Load }}$ is the normal load controlled by the deformation of the cantilever spring of a stage.

One more question for the researchers working in the cartilage mechanics area is a tribology of such a complicated structure. The interstitial fluid plays a role of lubricant in a biojoint and affects greatly tribological properties of the cartilage. Therefore experiments for obtaining relatively realistic quantitative magnitudes of friction coefficient are more preferable to be carried out in situ. Determination of tribological properties requires development of a plant prototype for friction measurements presented below.

The prototype is based on the atomic force microscope system. The model consists of a holder for AFM with stiffly fastened copper cantilever. On a free end of the cantilever a steel ball is fixed by an epoxy adhesive.

The AFM NT-206 allows implementing a collect backward data function. The idea of friction determination is to measure the cantilever twist angle around a longitudinal axis under the friction forces between the ball indenter and cartilage surface. The twist angle determination enables to estimate friction force and coefficient when additional calibration of cantilever deflection to force units is done. For the experiment deflection and torsion ranges of laser point 


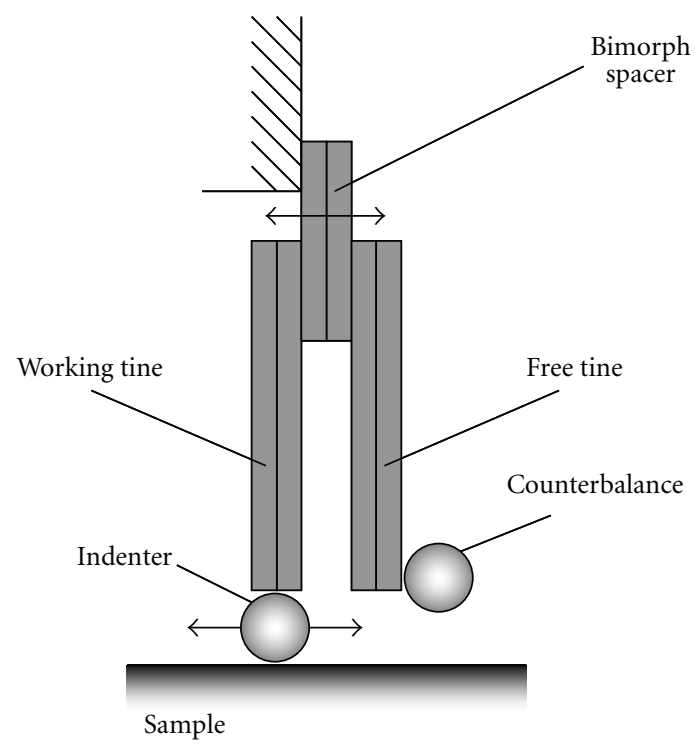

FIGURE 3: Oscillating tribometer.

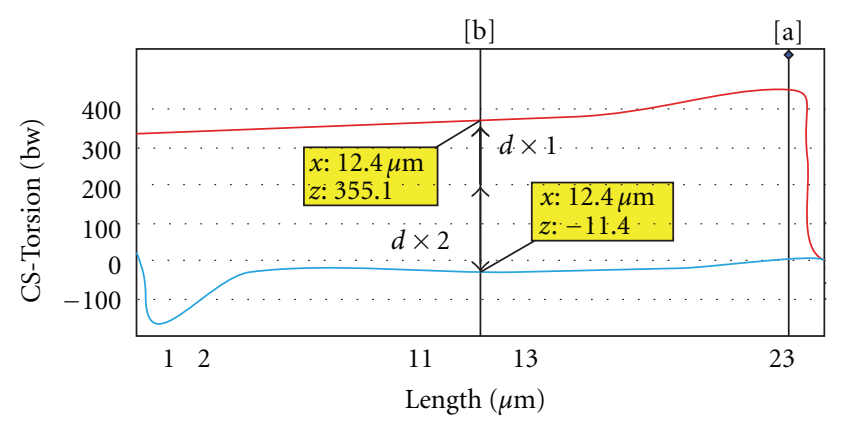

Absolute values:

[a] $x=23.4 \mu \mathrm{m} ; z(1)=440.5$ (null); $z(2)=20.1$ (null)

[b] $x=12.4 \mu \mathrm{m} ; z(1)=355.1$ (null); $z(2)=-11.4($ null $)$

Difference between markers:

$d x=11 \mu \mathrm{m} ; d z(10=35.4($ null $) ; d z 92)=31.5($ null $)$

FIgURE 4: Typical mode for the lateral friction force determination results.

are set to be equal. A cantilever twisting value $\mathrm{dZ}$ is obtained by recording the twist angle while scanning the specimen in forward and reverse directions and dividing a disparity in two. Thereby the friction force is

$$
F_{\mathrm{fr}}=\frac{d Z \cdot r \cdot G \cdot h^{3} \cdot b}{\left(l^{2} \cdot s\right)} .
$$

Here $G$ is the shear modulus for the cantilever material, $\mathrm{Pa} ; l$ is the cantilever length, $\mathrm{m} ; h$ is the cantilever thickness, $\mathrm{m} ; b$ is the cantilever width, $\mathrm{m}$.

\section{Results and Discussion}

3.1. Elastic Properties of the Cartilage. Figure 5 shows results of the study of topography structures on sample surface with the help of SEM and AFM. On the general relief one can
TABLE 1: Young's modulus values for joint cartilage under stretching.

\begin{tabular}{lcc}
\hline Elastic modulus & Value, $\mathrm{MPa}$ & References \\
\hline \multirow{2}{*}{ Short-term $E_{0}$} & 12,2 & {$[14]$} \\
& 2,35 & {$[12]$} \\
\hline \multirow{2}{*}{ Equilibrium $E_{¥}$} & 7,24 & {$[14]$} \\
& 0,71 & {$[12]$} \\
& 3,67 & {$[15]$} \\
\hline
\end{tabular}

distinctly see lacunes being bedding locations of cartilage cells (chondrocytes). SEM micrographs give a panoramic view of structure surface and layers of cartilage. AFM images allow to distinguish separate collagen fibrils and to measure their height and lateral dimensions. For the cross section marked on the AFM image (a), the fiber diameter reaches $100 \mathrm{~nm}$. Results of AFM measurement essentially repeat the ones obtained by SEM.

On the sliding surface of entire cartilage we can see closely arranged bundles of collagen fibers oriented parallel to the cartilage surface. Arrangement of the fiber bundles forms the joint cartilage surface relief that has regular waviness. It can be mentioned that surface of radial sections also demonstrates fibrous oriented structure with more tight bedding of chondrocytes, that is, lacunes. Surface of tangential section significantly differs from the previous cases. Background relief does not find explicit collagen network and has more tight arrangement of the lacuna hollows. Such difference is quite expectable as surfaces of entire cartilage and its radial section are formed, according to the structure scheme, of collagen fibers arranged just in the plane of analyzed surfaces.

In the third case, fibers oriented in the section plane were not found. It is expectable for tangential slices to show cross sections of fibers and fibrils. Perhaps, high hollow density in this case is conditioned not only by chondrocyte lacunes but also by depressions surrounding the cut fibers.

As the result of micro- and nanoindentation data analysis, dependencies of Young's modulus $E$ on the indenter penetration depth $H$ were obtained. Figure 6 shows the results of entire cartilage indentation by nanoindenter (AFM tip) and microindenter (ball). Utmost depth of nanoindentations did not exceed $110 \mathrm{~nm}$, that one of microindentations reached $600 \mathrm{~nm}$.

Young's modulus in microscale varies in narrow range of $0.6-0.7 \mathrm{MPa}$. For nanoscale measurements, this value varies during the loading process almost for an order of magnitude from 1.8 to $0.5 \mathrm{MPa}$. In our opinion, the decrease of Young's modulus value during the loading is conditioned not so much by its change across the depth as by viscoelastic behavior of collagen fibers in surface layer since they, taking into account their arrangement, deform by deflection. Note that full cycle of loading in both cases was about $10 \mathrm{~s}$. The obtained quantitative values correspond well with known literature data on study of cartilage elasticity by stretching (Table 1).

In our case, Young's modulus was estimated using the procedure of contact deformation. It is found that cartilage 
(1) SEM data
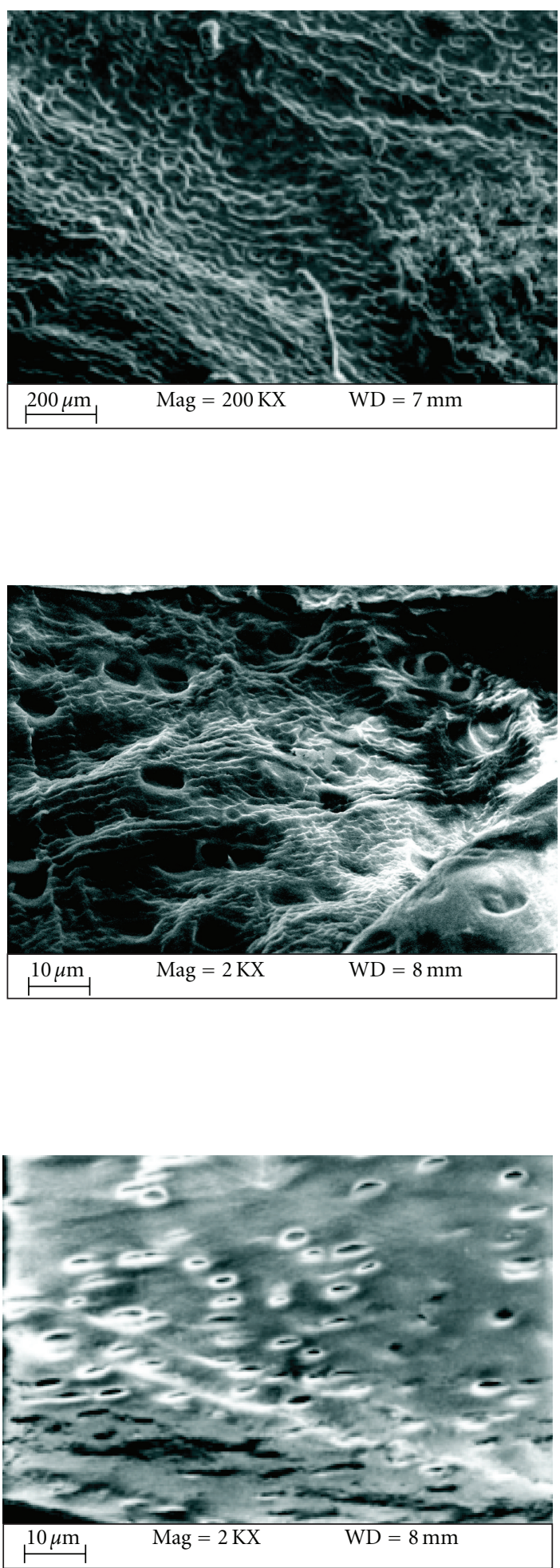

(2) AFM data

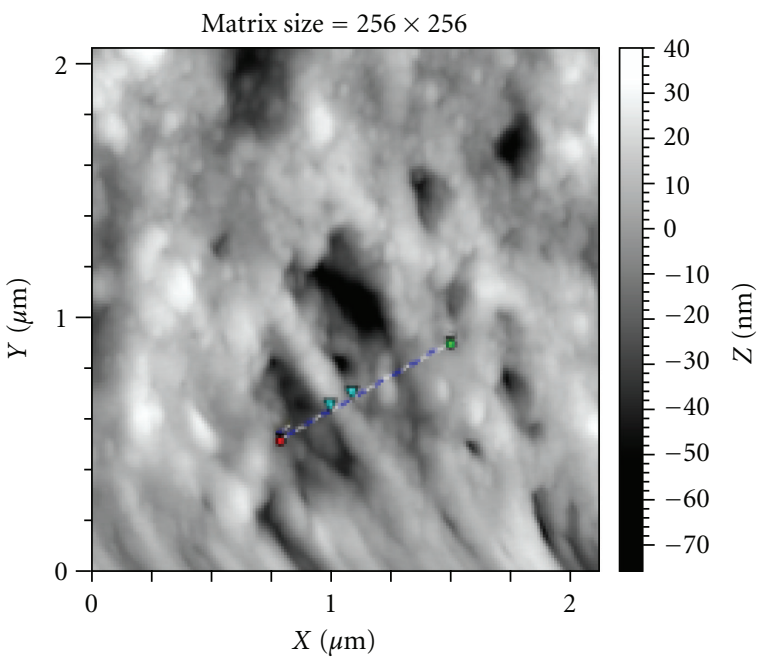

(a)

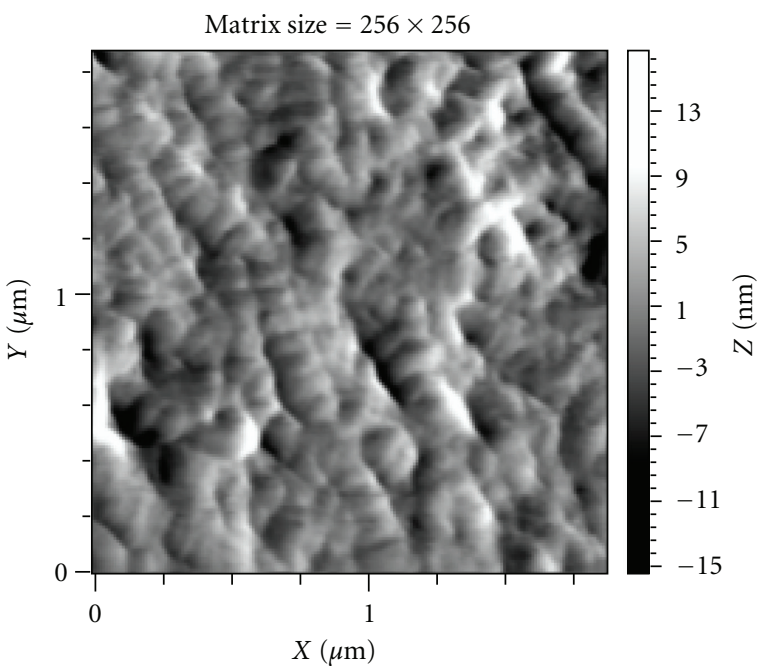

(b)

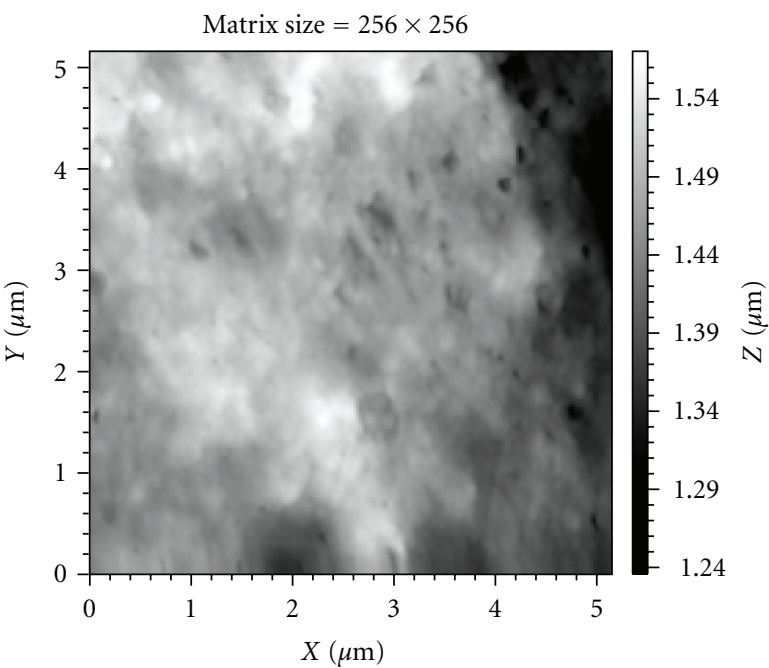

(c)

Figure 5: Results of the SEM (column 1) and AFM (column 2) studies of the sliced cartilage samples: (a) entire cartilage surface; (b) radial slice surface; (c) tangential slice surface. SEM images scale bars are: (a) 100 micron; (b) 10 micron; (c) 10 micron. AFM images scan sizes are: (a) $2.2 \times 2.2$ micron; (b) $1.7 \times 1.7$ micron; (c) $5.2 \times 5.2$ micron. 


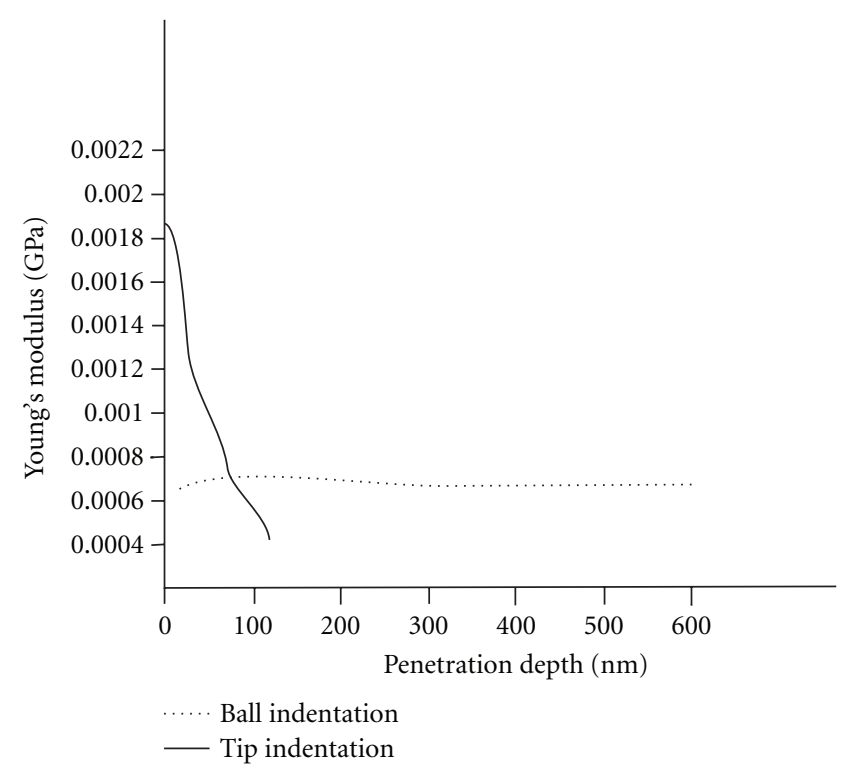

Figure 6: Young's modulus versus indenter penetration depth for the entire cartilage surface at different scale levels of measurement.

has weaker viscous behavior under contact loading on microlevel.

On microlevel, elastic reaction of cartilage on external contact load is a result of the cartilage composite structure functioning: collagen matrix-proteoglycans-water complex demonstrates extremely high bearing capacity without noticeable variation of the elasticity with time. Meanwhile nanoindenter tip allows us to estimate Young's modulus of separate components of natural cartilage, collagen fibers in most cases.

Similar comparison of cartilage deformation on microand nanoscale was carried out in the work. However, there is no common opinion for the situation. For example, in paper [16] the Young's modulus values on microlevel are $2.6 \pm$ $0.05 \mathrm{MPa}$. On nanolevel they were as follows: $E=0.027 \pm$ $0.003 \mathrm{MPa}$. Substantial difference from the case considered in our work is concluded in much smaller microindenter curvature radius [15] that was 2.5 microns. That did not allow to involve all composite structure of cartilage into the contact deformation.

Figure 7 gives data on comparison of nanoindentation results for cartilage slices of different orientation. As you can see, character of dependency of Young's modulus on penetration depth (i.e., on loading time) practically coincides for radial slices and entire cartilage. For both samples significant time dependence of elastic modulus was observed. And quantitative coincidence takes place at that. Young's modulus values for tangential slice in initial portion of the curve significantly differs from those ones for radial slice and whole cartilage in the same interval. Deformation character also significantly differs: tangential slice scarcely demonstrates behavior resembling viscoelastic deformation. Such difference can be explained by the fiber flexure in the first two cases as in the third case the deformation is directed along fiber axis. That is, Young's

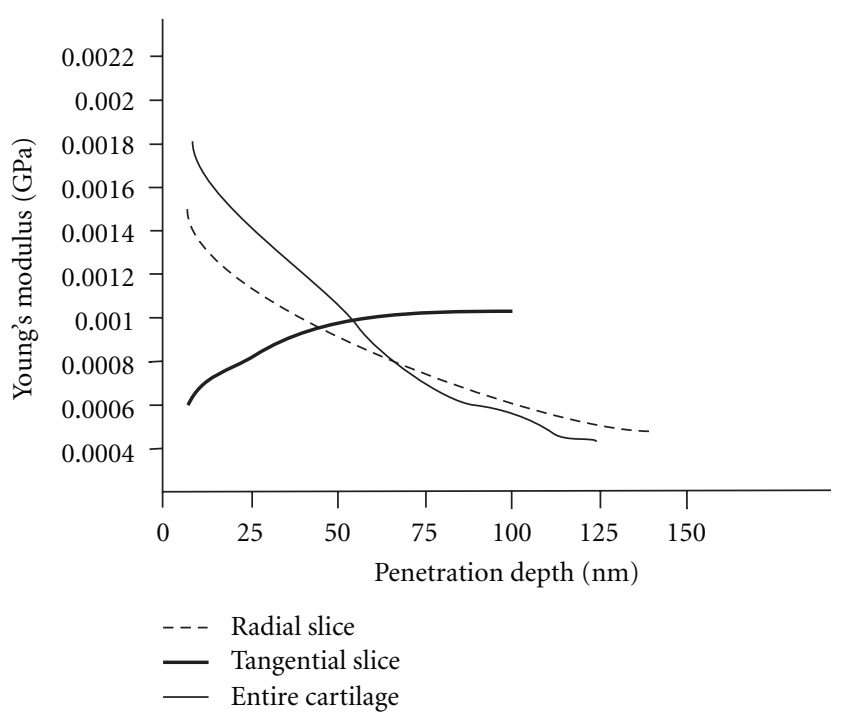

Figure 7: Young's modulus versus penetration depth of AFM tip for cartilage sliced in different directions.

modulus depends on direction of load application to fibers: tangential arrangement of collagen fibers provides more uniform distribution of external pressure over the cartilage surface; meanwhile, higher compliance is reached when collagen fibers sustain flexure.

3.2. Friction Measurements Using the Tuning Fork. In the work tribological properties of the natural block cartilage on microlevel were investigated using the tuning fork, and dependences of friction coefficient from a load (Figure 8) were drawn.

For the dependence of the friction coefficient from the applied load (Figure 8) each curve is numbered in the order of its recovery: curve 1 at the 3 rd minute of the experiment, curve 2 at the 30th minute, curve 3 at 45 th minute, curve 4 at 55 th minute and curve 5 at 65 th minute.

The maximums are observed (except curve 1), but for the curves 2,3 the maximum value increases, and at the same time for the curves 4,5 the maximum value falls down sharply.

At the beginning friction coefficient curve 1 does not achieve its maximum under such loads. It can be explained by relative wholeness. Collagen fibrils in the upper layer are tangentially located, and they redistribute loads evenly on the surface, therefore friction coefficient increases at the beginning of curve 1 and does not change by obtaining some definite value. Curves 2 and 3 are obtained at the 30th and 45th minutes, respectively, after the experiment beginning. During this time the cartilage became dried, the upper layer degraded, the collagen fibrils orientation changed, and the elasticity decreased. As consequence firstly the friction coefficient highly increases, then under a definite load the interstitial fluid is extruded, and friction coefficient sinks. For curves 4 and 5 considerable decline of the friction coefficient is observed. The occurrence is, probably, related to the failure 


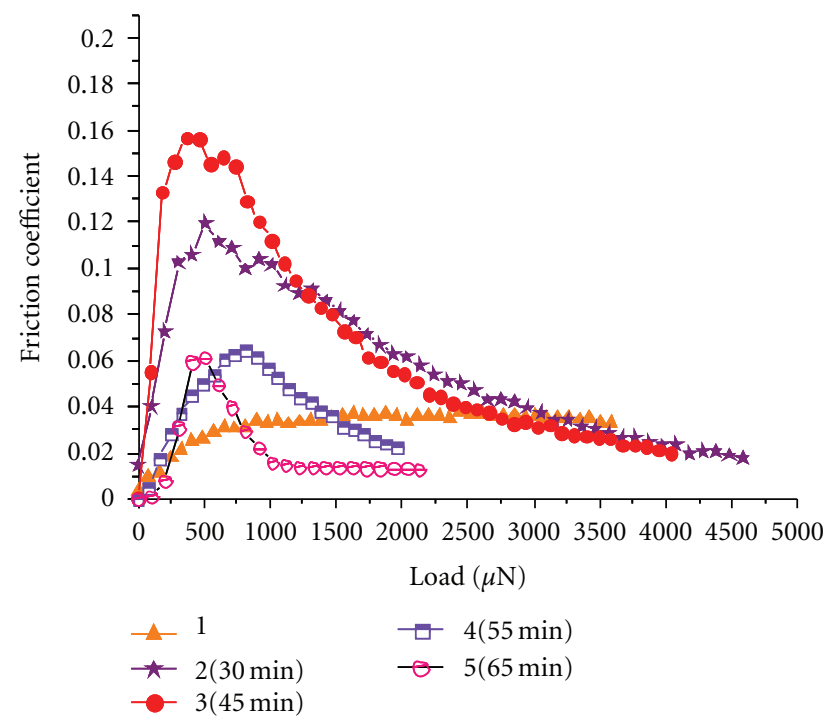

Figure 8: Dependences for friction coefficient of the bulk cartilage from the applied loads (curves are numbered in order of recovery; time passed from the beginning of the experiment is pointed in brackets).

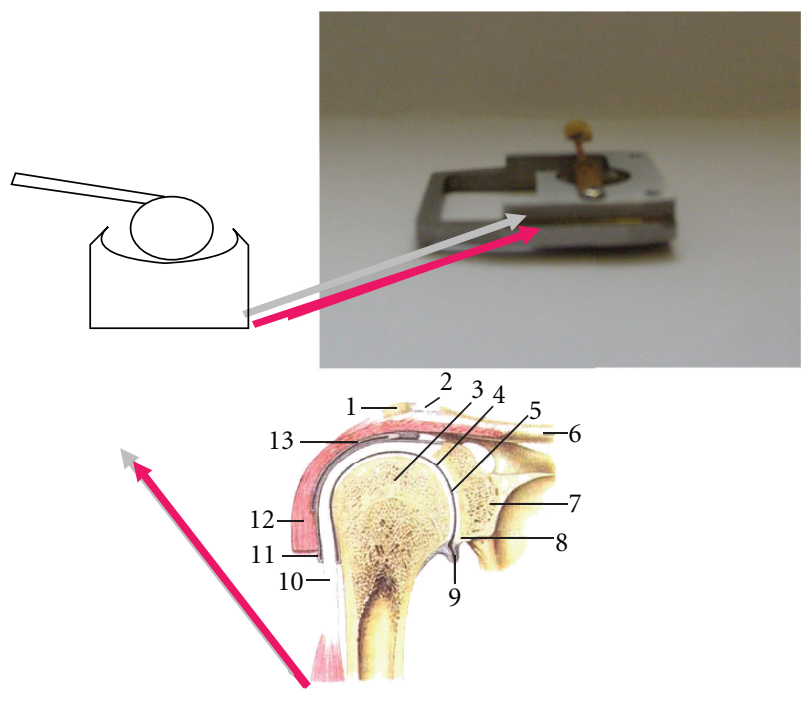

FIGURE 9: Friction measurement plant scheme.

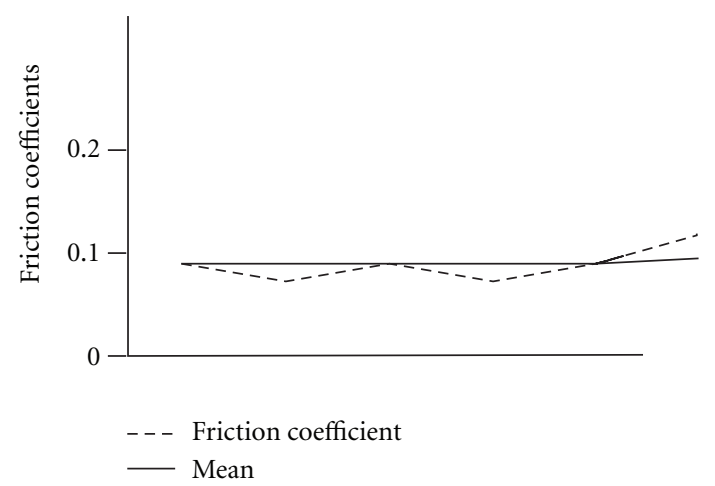

FIGURE 10: Results of an acetabulum friction investigation. of the degraded by the friction thin layer. New uncovered surface is much more elastic and smooth, with greater penetration of the inner fluid through the cartilage surface. Therefore even under the small loads the fluid extrusion from the cartilage bulk occurs, and the friction coefficient reduction is observed.

3.3. Friction Measurement Plant Prototype. The rat cartilage friction was also measured in the work. The presented prototype (Figure 9) can be adapted to the different AFM constructions. Depending on the explored material a replacement of the ball indenter for friction enables realizing. For next series of the experiment the steel ball was substituted by the biojoint part.

Friction coefficient calculation for the cartilage tissue reveals values of 0.08 (Figure 10). The results are influenced by the fact that the specimens were treated by an alcohol and also the great role was played by the interstitial fluid absence. Though the experiment demonstrates a potential of implementing such a technique for friction investigations in biojoints.

\section{Conclusion}

The conducted investigations of structure and elastic properties of cartilage on micro- and nanolevels showed that stable bearing capacity of the natural material reduced time dependence of their elastic properties reveals in case of involvement of all composite structure of cartilage tissue into the process of contact deformation and only when deformation direction coincides with that characteristic for natural conditions in joint. In this case contact pressure is redistributed more uniformly, and whole system of collagen matrix-proteoglycans-water is set in operation and impedes abrupt change of pressure and big deformations in the joint.

Collagen fibers under flexure show viscoelastic behavior and are unable to provide high mechanical properties characteristic to cartilage tissue when isolated and do not interact as a components of cartilage matrix filled with water.

This implies that only joint consideration of role of all cartilage structure components can help to explain its unique behavior with respect to the external load.

Influence of the interstational fluid presence on tribological properties of the cartilage was confirmed using the tuning fork and the collect backward data function of the AFM. First experiment of the implementation of the friction measurement plant consisting the biojoint part corroborated its own application outlook.

\section{References}

[1] E. M. Hasler, W. Herzog, J. Z. Wu, W. Müller, and U. Wyss, "Articular cartilage biomechanics: theoretical models, material properties, and biosynthetic response," Critical Reviews in Biomedical Engineering, vol. 27, no. 6, pp. 415-488, 2000.

[2] T. E. Hardingham, "Fell-Muir lecture: cartilage 2010-the known unknowns," International Journal of Experimental Pathology, vol. 91, no. 3, pp. 203-209, 2010. 
[3] M. Scherge, Bio-Micro-Nanotribology. Natural Solutions, Springer, New York, NY, USA, 2000.

[4] B. I. Kupchinov, S. F. Ermakov, and E. D. Beloenko, Bio Tribology of Synovial Joints, Vedy, Minsk, Belarus, 1997.

[5] K. C. H. Wierzcholski, "Friction forces for human hip joint lubrication at a naturally permeable cartilage," International Journal of Applied Mechanics and Engineering, vol. 11, no. 3, pp. 515-527, 2006.

[6] M. J. Kääb, K. Ito, J. M. Clark, and H. P. Notzli, “Deformation of articular cartilage collagen structure under static and cyclic loading," Journal of Orthopaedic Research, vol. 16, no. 6, pp. 743-751, 1998.

[7] M. J. Kääb, K. Ito, B. Rahn, J. M. Clark, and H. P. Nötzli, “Effect of mechanical load on articular cartilage collagen structure: a scanning electron-microscopic study," Cells Tissues Organs, vol. 167, no. 2-3, pp. 106-120, 2000.

[8] J. Töyräs, T. Lyyra-Laitinen, M. Niinimäki et al., "Estimation of the Young's modulus of articular cartilage using an arthroscopic indentation instrument and ultrasonic measurement of tissue thickness," Journal of Biomechanics, vol. 34, no. 2, pp. 251-256, 2001.

[9] J. P. A. Arokoski, J. S. Jurvelin, U. Väätäinen, and H. J. Helminen, "Normal and pathological adaptations of articular cartilage to joint loading," Scandinavian Journal of Medicine and Science in Sports, vol. 10, no. 4, pp. 186-198, 2000.

[10] M. L. Schenker, R. Martin, D. E. Weiland, and M. J. Philippon, "Current trends in hip arthroscopy: a review of injury diagnosis, techniques, and outcome scoring," Current Opinion in Orthopaedics, vol. 16, no. 2, pp. 89-94, 2005.

[11] T. Franz, E. M. Hasler, R. Hagg, C. Weiler, R. P. Jakob, and P. Mainil-Varlet, "In situ compressive stiffness, biochemical composition, and structural integrity of articular cartilage of the human knee joint," Osteoarthritis and Cartilage, vol. 9, no. 6, pp. 582-592, 2001.

[12] F. C. Linn, "Lubrication of animal joints. I. The arthrotripsometer," Journal of Bone and Joint Surgery A, vol. 49, no. 6, pp. 1079-1098, 1967.

[13] http://microtm.com/download/manual-nt206e2004.doc.

[14] N. Burnham, "Measuring the nanomechanical properties and surface forces of materials using an atomic force microscope," Journal of Vacuum Science \& Technology A, vol. 7, pp. 29062913, 1989.

[15] B. Bhusha, H. Fuchs, and S. Hosaka, Applied Scanning Probe Methods, Springer, New York, NY, USA, 2002.

[16] M. Stolz, R. Raiteri, A. U. Daniels, M. R. VanLandingham, W. Baschong, and U. Aebi, "Dynamic elastic modulus of porcine articular cartilage determined at two different levels of tissue organization by indentation-type atomic force microscopy," Biophysical Journal, vol. 86, no. 5, pp. 3269-3283, 2004. 

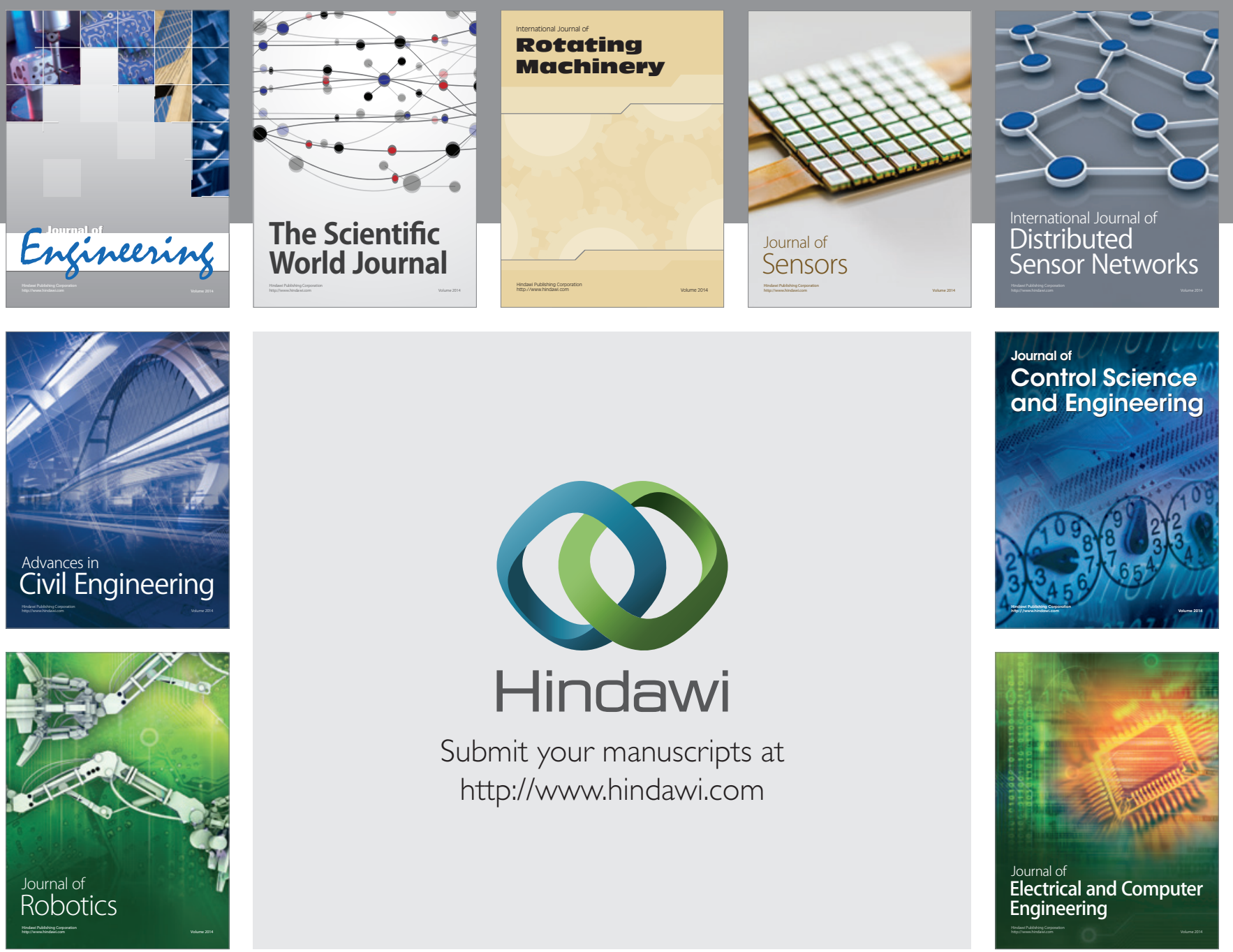

Submit your manuscripts at

http://www.hindawi.com
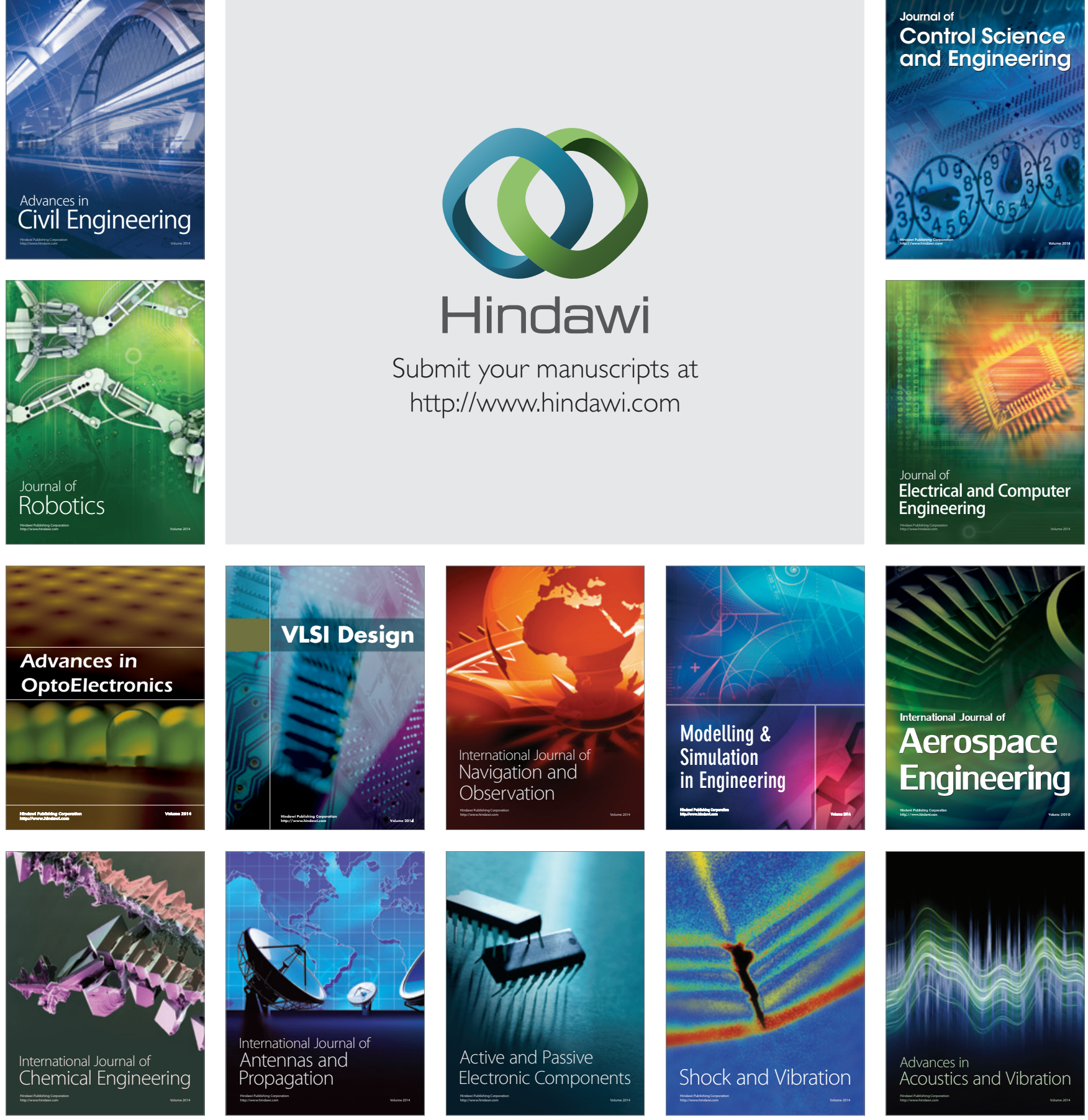